Research Article

\title{
Well-defined sub-nanometer graphene ribbons synthesized inside carbon nanotubes
}

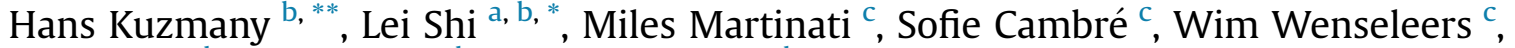 \\ Jenő Kürti ${ }^{d}$, János Koltai ${ }^{d}$, Gergő Kukucska ${ }^{d}$, Kecheng Cao ${ }^{\text {e }}$, Ute Kaiser ${ }^{\text {e }}$, Takeshi Saito ${ }^{\text {f, }}$ \\ Thomas Pichler ${ }^{\mathrm{b}}$ \\ a School of Materials Science and Engineering, State Key Laboratory of Optoelectronic Materials and Technologies, Nanotechnology Research Center, Sun \\ Yat-sen University, Guangzhou, 510275, Guangdong, PR China \\ ${ }^{\mathrm{b}}$ Faculty of Physics, University of Vienna, 1090, Wien, Austria

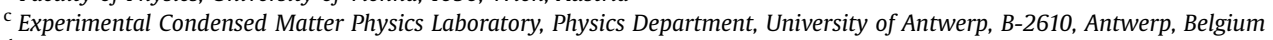 \\ ${ }^{d}$ Department of Biological Physics, ELTE Eötvös Loránd University, Pázmány Péter Stny. 1/A, 1117, Budapest, Hungary \\ e Central Facility for Electron Microscopy, Electron Microscopy Group of Materials Science, Ulm University, Ulm, 89081, Germany \\ ${ }^{\mathrm{f}}$ Nanomaterials Research Institute, National Institute of Advanced Industrial Science and Technology (AIST), 305-8565, Tsukuba, Japan
}

\section{A R T I C L E I N F O}

\section{Article history:}

Received 22 June 2020

Received in revised form

21 August 2020

Accepted 27 August 2020

Available online 6 September 2020

\section{Keywords:}

Graphene nanoribbons

Electronic structure

Raman scattering

Resonance profiles

Albrecht theory

GW calculation

\begin{abstract}
A B S T R A C T
Graphene nanoribbons with sub-nanometer widths are extremely interesting for nanoscale electronics and devices as they combine the unusual transport properties of graphene with the opening of a band gap due to quantum confinement in the lateral dimension. Strong research efforts are presently paid to grow such nanoribbons. Here we show the synthesis of 6- and 7-armchair graphene nanoribbons, with widths of 0.61 and $0.74 \mathrm{~nm}$, and excitonic gaps of 1.83 and $2.18 \mathrm{eV}$, by high-temperature vacuum annealing of ferrocene molecules inside single-walled carbon nanotubes. The growth of the so-obtained graphene nanoribbons is evidenced from atomic resolution electron microscopy, while their welldefined structure is identified by a combination of an extensive wavelength-dependent Raman scattering characterization and quantum-chemical calculations. These findings enable a facile and scalable approach leading to the controlled growth and detailed analysis of well-defined sub-nanometer graphene nanoribbons.
\end{abstract}

(C) 2020 Elsevier Ltd. All rights reserved.

\section{Introduction}

Sub-nanometer graphene ribbons (graphene nanoribbons, GNRs) are promising structures for future electronic devices [1,2] as they are considered of unifying the unique electronic properties of graphene [3] with a reasonably sized gap in their electronic structure. The gap results from quantum confinement in the lateral direction and consequently scales with the inverse width of the ribbons [4,5]. The ribbons are strips of carbon atoms cut out from a graphene lattice. At present most common strips are of the armchair type, i.e., the edge of the ribbons consists of coaxial

\footnotetext{
* Corresponding author. School of Materials Science and Engineering, State Key Laboratory of Optoelectronic Materials and Technologies, Nanotechnology Research Center, Sun Yat-sen University, Guangzhou, 510275, Guangdong, PR China.

** Corresponding author.

E-mail addresses: hans.kuzmany@univie.ac.at (H. Kuzmany), shilei26@mail.sysu. edu.cn (L. Shi).
}

carbon pairs oriented parallel to the direction of the ribbon axis (armchair graphene nanoribbons (AGNR)). They are characterized by the number $n$ of such pairs across their width. The electronic structures of AGNR can be classified into $n=3 p, 3 p+1$ and $3 p+2$ species, where $\mathrm{p}$ is an integer [4]. One of the most commonly investigated structures is the $\mathrm{n}=7$ AGNR (7-AGNR) [6,7] with band gaps between 2.1 and $2.3 \mathrm{eV}$ as reported from scanning tunneling spectroscopy (STS) and optical studies [8]. More recently ribbons with more complex topologies were grown which have coved zigzag, chevron or chiral type structures $[7,9,10]$. The ribbons are usually grown on Au substrates from preselected and properly designed flat poly-aromatic hydrocarbon molecules (PAHs) [11]. In this case the PAHs are vacuum deposited on the substrate and subsequently transformed to polymeric units with nanoribbon structure. To take advantage of the ribbons grown in this way subsequent transformation to insulating substrates is necessary [12]. Raman scattering was used to evidence that the nanoribbons do not suffer in quality by this transformation. 
In general the carbons at the edge of the ribbons are saturated by hydrogen but by selecting special PAHs, bandgap engineering [13-15], construction of ribbon heterojunctions [16,17] and ribbons with unusual electronic properties [18] have been demonstrated where topological properties of the ribbons under investigation play an important role. Most recently ribbons became relevant for applications in photocatalytic hydrogen generation [19].

Besides STS and electron microscopy, Raman scattering has repeatedly been used to characterize GNR. Several Raman active vibrational modes were identified to characterize the ribbons. Such modes are among others the radial breathing like mode (RBLM), the $\mathrm{CH}$ in plane bending mode $(\mathrm{CH}-\mathrm{ipb})$, the $\mathrm{D}$ line, and the graphene $\mathrm{G}$ line $[12,13]$. The RBLM frequency scales with the square root of the inverse ribbon width [20] or with the inverse ribbon width [21], and is the pendant to the radial breathing mode of the carbon nanotubes.

Filling and consecutive chemical reactions inside single-walled carbon nanotubes (SWCNTs) is a promising technique to grow new nanoscale materials in general [22-24]. In addition, the onedimensional geometry of the CNTs is an excellent template for the controlled growth of conventional or exotic low-dimensional compounds [25-27]. Here, we show that this in-tube synthesis can be used to grow GNRs with well-defined sub-nanometer width. Growth of such narrow ribbons with controlled width has previously been limited to the polymerization of specific PAHs on $\mathrm{Au}$ substrates [28]. Although providing well-defined freestanding ribbons with relatively high yield, the flexibility of preparing GNRs with different widths and structures is limited by the availability of appropriate precursor molecules. When synthesizing GNRs inside SWCNTs, the width of the GNRs is determined by the diameter of the SWCNTs, hence could in the future be flexibly tuned by starting from CNT samples with different diameters (eventually even diameter/chirality-sorted SWCNTs). Moreover, the hybrid structures of encapsulated ribbons and a semiconducting SWCNT, both with a different band gap can be interesting also from a materials design perspective.

The growth of GNRs inside SWCNTs has been demonstrated by first filling the SWCNTs with flat precursor molecules, such as coronene [29,30] or other PAHs [28], and subsequently transforming them at elevated temperature. Although these initial results were very promising, growth of high-quality specific types of GNRs with well-defined widths still remained a challenge. In particular the analysis of the electronic properties of the objects inside the tubes remained difficult due to the overlapping electronic transitions of the carbon nanotubes themselves. To avoid this overlap, functionalization was necessary of the otherwise pristine tubes [31]. As it will be demonstrated below, Raman scattering, in particular in combination with evaluation of resonance Raman excitation profiles, is an excellent tool to reveal the electronic structure of the objects inside the tubes. This is due to the double selective nature of the resonance Raman scattering process. It is selective with respect to the geometrical structure of the objects by the vibrational mode but also selective with respect to the electronic structure by the resonance profile. Small width of the Raman lines indicates well-defined geometrical structures and sharp excitation profiles provide evidence for uniform electronic configuration of the GNRs.

In this work, we demonstrate the synthesis of two specific GNRs, i.e., 6- and 7-AGNRs, with well-defined geometrical structure, from the bulky molecule ferrocene $\left(\mathrm{FeC}_{2}\right)$. Evidence for the growth of the AGNRs inside the tubes comes from aberration-corrected highresolution transmission electron microscopy (AC-HRTEM) and from Raman scattering. We also show that wavelength-dependent Raman spectroscopy gives direct access to the electronic and optical properties of the encapsulated objects by the evaluation of resonance Raman excitation profiles. This allows determining experimentally the excitonic gap and even more the electronic structure beyond the gap. High level first principle calculations provide for the first-time relative intensities of the Raman lines of the GNRs by using the Placzek formulation with energy dependent polarizabilities [32]. The latter procedure is a fundamental progress in the evaluation of Raman intensities.

\section{Experimental results and analysis}

Aberration-Corrected High Resolution Transmission Electron Microscopy (AC-HRTEM)The standard feature for the observation of encapsulated ribbons in TEM is an alternating pattern of narrow and wide signals from the material inside $[28,33]$. It originates from an electron beam induced twisting of parts of the ribbons. Fig. 1 depicts a collection of results from $\mathrm{FeCp}_{2}$ filled and subsequently transformed SWCNT species as acquired by the SALVE instrument with $\mathrm{Cs} / \mathrm{Cc}$ aberration corrector. It demonstrates the twisting of the graphene ribbons by the electron beam (panel a-e) and provides images of the ribbons in atomic resolution with corresponding simulations (panel $\mathrm{f}-\mathrm{j}$ ). The time-dependent AC-HRTEM images show clearly that the observed species are flat objects, that twist under the influence of the electron beam, and cannot be identified as inner nanotubes. The AC-HRTEM images (panels $f-j$ ) provide clear evidence of the atomic structure of the ribbons, however, also demonstrate that the nanoribbons are very unstable under the electron beam and defects along the length of the structure are quickly generated by the beam. Therefore, these AC-HRTEM images are not representative for the quality of the as-grown ribbons and only serve to evidence that the structures are flat ribbons. Evidence for the well-defined electronic structure of the as-grown ribbons comes from our detailed wavelength-dependent Raman spectroscopic experiments (see below). More details on the AC-HRTEM are provided in the Supporting Information Section (a).

Raman Scattering Previously, we reported the observation of a set of Raman lines after thermal conversion of $\mathrm{FeCp}_{2}$ filled SWCNT $[24,34]$ but the origin of these lines remained unclear as they did not fit to proper model calculations and high resolution TEM was not available. Here we identified the origin of the Raman lines which turned out to be very sensitive to transformation temperature. Fig. 2a-d depicts this behavior in a plot where Raman intensities are characterized by a color code as a function of Raman frequency and transformation temperature. Raman spectra were normalized to the $2 \mathrm{D}$ band of the encapsulating tubes, which is least influenced by the changes induced by the GNR encapsulation. Fig. $2 \mathrm{a}$ and $\mathrm{b}$ depicts the responses measured for $568 \mathrm{~nm}$ excitation while Fig. 2c and d presents those for $633 \mathrm{~nm}$ excitation. At low temperature, only the RBMs (around $200 \mathrm{~cm}^{-1}$ ) and the G-line of the SWCNTs (around $1600 \mathrm{~cm}^{-1}$ ) are observed. When increasing the transformation temperature beyond $500{ }^{\circ} \mathrm{C}$, depending on the excitation laser used two groups of Raman lines can be observed. They exhibit a maximum Raman intensity between 600 and $700{ }^{\circ} \mathrm{C}$, indicated by the horizontal white arrows in the figure. As shown further on, these two groups of lines correspond to the in-tube synthesis of AGNRs with different widths.

The lower panels of Fig. 2 depict the Raman spectra explicitly. Panels e and $\mathrm{f}$ are spectra recorded at $568 \mathrm{~nm}$ (transformation temperature $600{ }^{\circ} \mathrm{C}$ ) and $\mathrm{g}$ and $\mathrm{h}$ are spectra recorded at $633 \mathrm{~nm}$ (transformation temperature $700{ }^{\circ} \mathrm{C}$ ), for two different Raman frequency regions. When comparing the Raman spectra of the ferrocene-filled and temperature-transformed samples (red) with the spectra of filled SWCNTs without transformation (black), the new Raman features appear very well separated from the response of the SWCNTs in general. In the high-frequency region, the spectra of the SWCNTs can be subtracted straight forwardly, yielding the 

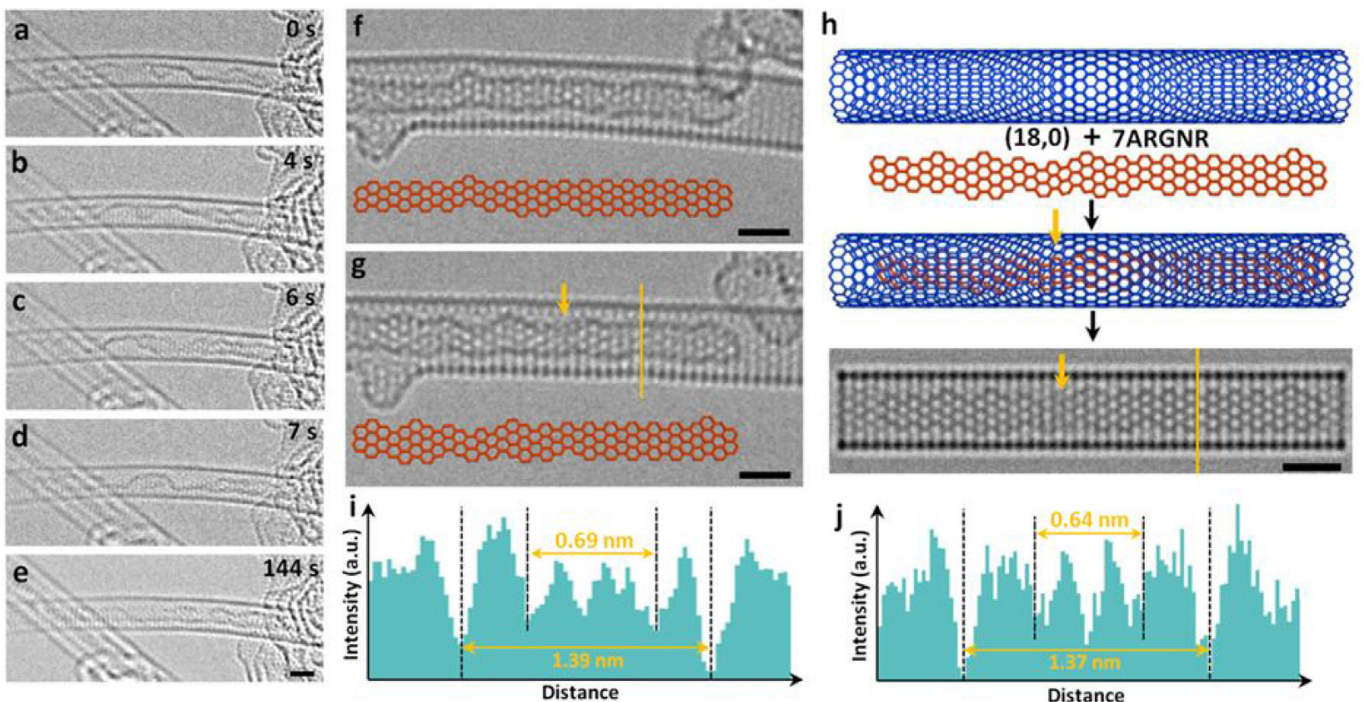

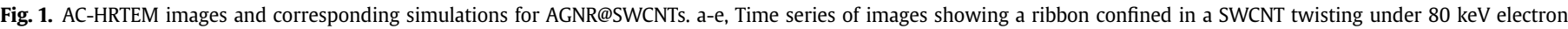

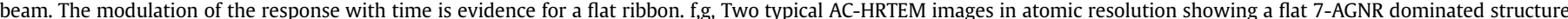

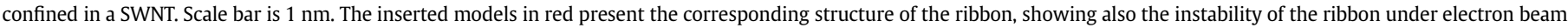

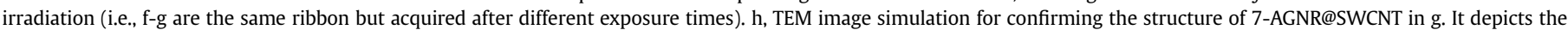

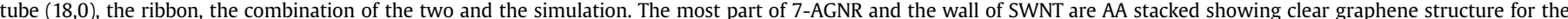

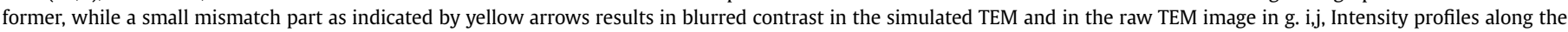

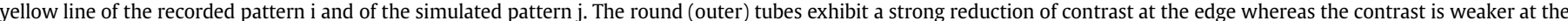

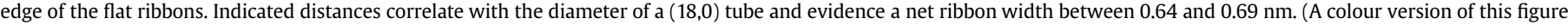
can be viewed online.)
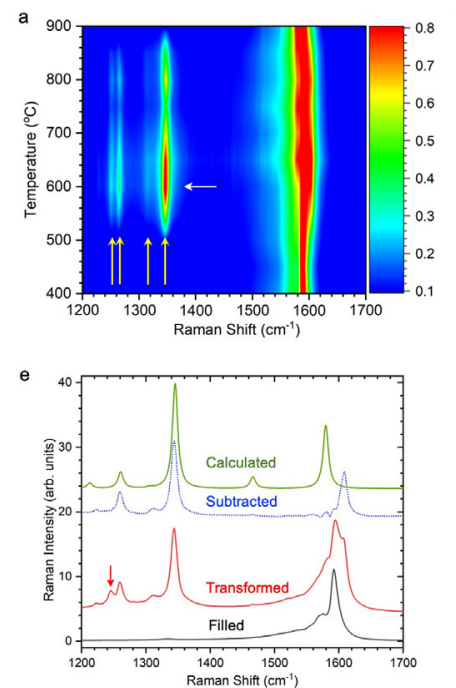
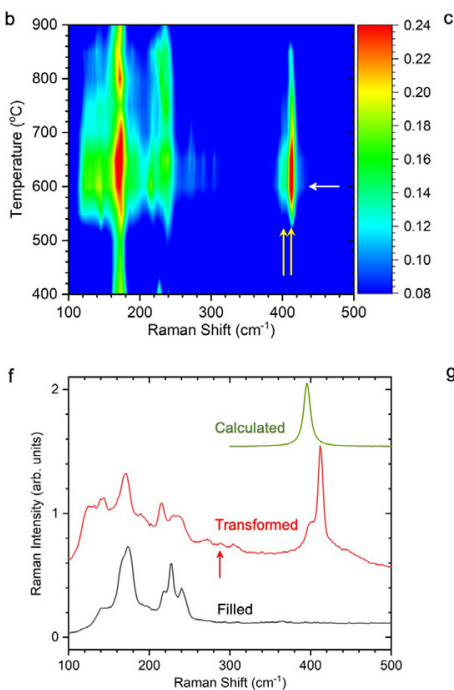
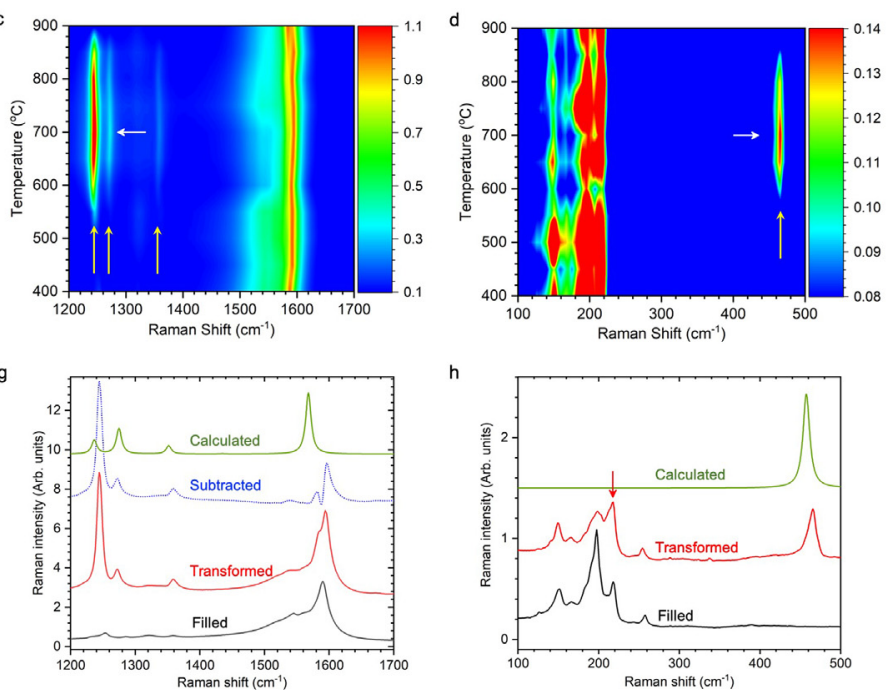

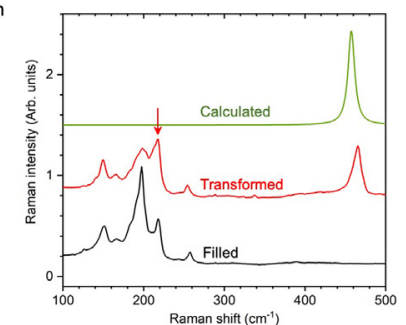

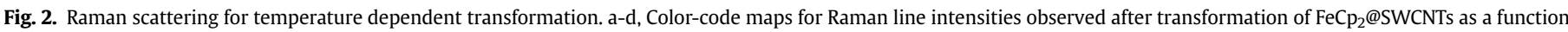

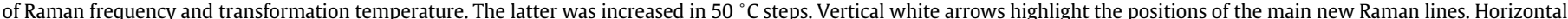

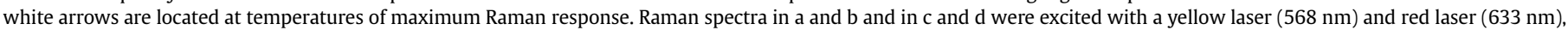

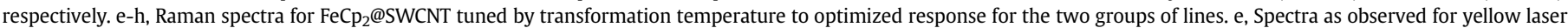

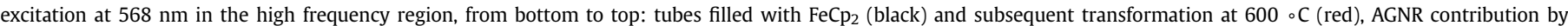

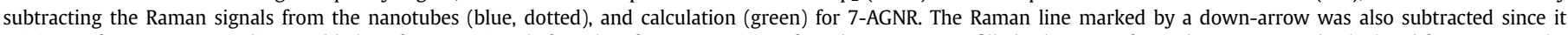

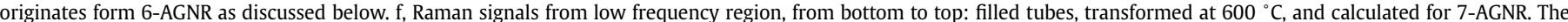

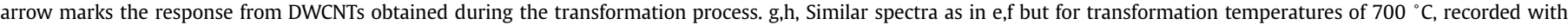
$633 \mathrm{~nm}$ laser, and as calculated for 6-AGNR. (A colour version of this figure can be viewed online.)

Raman response of the newly synthesized objects (blue dotted spectra in panels e and g). For the low-frequency region, a subtraction is more difficult, since during the transformation also inner tubes are formed thereby changing the response of the SWCNTs as well (red arrows). Even though, due to their higher frequency, the lines from the RBLMs of the GNRs can be well separated from the response of the CNTs. The figures demonstrate very narrow line widths of the order of $10 \mathrm{~cm}^{-1}$ for the response of the nanoribbons. This linewidth is equivalent to the Raman response of high-quality ribbons grown on Au substrates and subsequently transferred to semiconducting substrates $[2,12,35]$. Such narrow line widths (in combination with a clear resonance profile) can only be observed 
for nanoribbons with a well-defined width along their length, as width variations would lead to inhomogeneous broadening of the lines or even disappearance of the RBLM mode. Fig. 2 also presents the calculated Raman spectra for 7-AGNR species (panels e and f, in green) and for 6-AGNR (panels $g$ and $h$, in green), which were obtained from first principle calculations using the dynamical Placzek formalism [32]. More details about these calculations are in the Method section and in the Supporting Information Sections (b). They correspond very well to the observed experimental lines, even in relative intensity. A blown up version of this comparison can be found in the Supporting Information Section (d). For the 7-AGNR, the high-frequency modes are accordingly identified as the $\mathrm{CH}$-ipb mode $\left(1258 \mathrm{~cm}^{-1}\right)$, the $\mathrm{D}$-line $\left(1344 \mathrm{~cm}^{-1}\right)$ and the $G$ line $\left(1606 \mathrm{~cm}^{-1}\right.$ ). The low frequency mode at $414 \mathrm{~cm}^{-1}$ (with small shoulder at $400 \mathrm{~cm}^{-1}$ ) can be identified as the RBLM. Likewise, for the 6-AGNR the RBLM is located at $465 \mathrm{~cm}^{-1}$, the $\mathrm{CH}$-ipb mode at $1243 \mathrm{~cm}^{-1}$, the $\mathrm{D}-$ line at $1358 \mathrm{~cm}^{-1}$ and the $G$ line at $1595 \mathrm{~cm}^{-1}$. Note that the latter is strongly overlapping with the $G$ line of the SWCNTs which prevents its direct determination from the difference (blue) spectra. However, the frequency can be obtained by measuring the sum of the $\mathrm{CH}$-ipb and the $\mathrm{G}$ line of the ribbons which is observed at $2839 \mathrm{~cm}^{-1}$. This localizes the $G$ line at $1595 \mathrm{~cm}^{-1}$.

Table 1 presents the experimental Raman frequencies and line widths for both the 6-AGNR and 7-AGNR and compares it to our theoretical values as well as to values for the same modes measured on Au-substrates for 7-AGNR [6]. For the 6-AGNR a comparison is made with the only reported data originating from the fusion of linear chains of poly-paraphyenlye at $800 \mathrm{~K}$ [36]. The agreement between calculated and experimentally determined Raman frequencies in the high-frequency region is better than $2 \%$ and also the agreement between calculated and experimental relative line intensities is excellent, except for the $\mathrm{CH}$-ipb of the 6-AGNR which appears too weak in the calculation. The table highlights the unusually narrow width of the Raman lines from the encapsulated species. In three very recent reports, Raman spectra for 7-AGNR grown on Au substrates and subsequently transferred to transparent substrates are shown to exhibit similar narrow line widths as observed here [2,12,35].

Raman Excitation Profiles Raman excitation profiles were measured for all Raman lines in the low and in the high frequency

\section{Table 1}

Raman lines of 7-AGNR and 6-AGNR; Frequencies $\left(\omega_{p h}\right)$, intensities (weak (w), medium (m), strong (s), very strong (vs)), and linewidths W (FWHM, in parentheses) for the observed Raman lines (column Exp.) as compared to calculation (column Calc.) and to reference (column Ref.). For 7-AGNR and 6-AGNR the latter are from Refs. [6] and reference [36], respectively. The column with the calculated frequencies depicts also the difference to the experiment in \%. The last two columns depict the excited state frequencies $\left(\omega_{p h}^{*}\right)$ and the Huang-Rhys factor (HR) for the first excited state, both obtained from a fit of the resonances to the Albrecht A-term. All frequencies are given in $\mathrm{cm}^{-1}$ and rounded to integer values.

\begin{tabular}{|c|c|c|c|c|c|}
\hline Mode & $\begin{array}{l}\omega_{p h}(\mathrm{~W}) \\
\text { Exp. } \\
\left(\mathrm{cm}^{-1}\right)\end{array}$ & $\begin{array}{l}\omega_{p h} \\
\text { Calc.; \% } \\
\left(\mathrm{cm}^{-1}\right)\end{array}$ & $\begin{array}{l}\omega_{p h}(\mathrm{~W}) \\
\text { Ref. }[6,36] \\
\left(\mathrm{cm}^{-1}\right)\end{array}$ & $\begin{array}{l}\omega_{p h}{ }^{*} \\
\text { from fit } \\
\left(\mathrm{cm}^{-1}\right)\end{array}$ & $\begin{array}{l}\text { HR } \\
\text { from fit } \\
\left(\mathrm{cm}^{-1}\right)\end{array}$ \\
\hline \multicolumn{6}{|l|}{ 7-AGNR } \\
\hline RBLM & $414 \mathrm{~m}(7.5)$ & $395 \mathrm{~m} ; 4.5$ & $395 \mathrm{~m}(22.1)$ & 434 & 0.08 \\
\hline $\mathrm{CH}$-ipb & 1258 s (9.6) & $1261 \mathrm{~s} ; 0.2$ & $1263 \mathrm{~s}(30)$ & 1195 & 0.25 \\
\hline $\mathrm{D}$ & 1342 vs (10.8) & 1345 vs; 0.2 & 1344 vs (26) & 1275 & 0.25 \\
\hline G & $1607 \mathrm{~s}(13)$ & $1580 \mathrm{~s} ; 1.7$ & 1607 vs (31) & no fit & \\
\hline \multicolumn{6}{|l|}{ 6-AGNR } \\
\hline RBLM & $466 \mathrm{~m}(8)$ & $457 \mathrm{~m} ; 1.7$ & not reported & & \\
\hline \multirow[t]{2}{*}{$\mathrm{CH}$-ipb } & 1243 vs (9.6) & $1236 \mathrm{~m} ; 0.6$ & $1245 \mathrm{~s}(100)$ & 1281 & 0.17 \\
\hline & $1272 \mathrm{~m}(10.6)$ & $1272 \mathrm{~s} ; 0$ & & 1272 & 0.25 \\
\hline $\mathrm{D}$ & $1358 \mathrm{~m}(11.4)$ & $1352 \mathrm{w} ; 0.4$ & $1315 \mathrm{~m}$ & 1358 & 0.25 \\
\hline G & 1595 & $1568 \mathrm{~s} ; 1.7$ & $1590 \mathrm{vs}^{12}$ & no fit & \\
\hline
\end{tabular}

region for the excitation wavelength range from 400 to $800 \mathrm{~nm}$ with $5 \mathrm{~nm}$ steps. Fig. 3a shows overall results in the form of a twodimensional Raman map for a sample transformed at a temperature of $850{ }^{\circ} \mathrm{C}$.

Raman map means Raman intensities are plotted on a color code versus Raman shift and excitation energy. The procedure to create the Raman map from the experiments and to extract the excitation profiles for the various modes by wavelength-dependent fitting of the experimental data, is described in detail in the Supporting Information Section (e). The features between 1.7 and $2.1 \mathrm{eV}$ excitation in the lower part of the map (R1 and R4) correspond to the 6AGNR. The upper part has the resonances of the 7-AGNR (R2 and $\mathrm{R} 5$ ). $\mathrm{R} 3$ is the resonance of a weak and as yet unknown new line to be discussed below.

For a selected number of modes such as the $\mathrm{CH}$-ipb and the $\mathrm{D}$ line, experimental resonance profiles are plotted in Fig. $3 \mathrm{~b}$. The profiles for the 6-AGNR show only two vibronic peaks. In contrast, the resonances for 7-AGNR exhibit a highly structured profile which turned out to originate from vibronic sidebands as well as from higher exciton transitions.

The red lines in Fig. 3b are fits from the Kramers-HeisenbergDirac (KHD) theory for resonance Raman scattering [37,38] with wave functions in the adiabatic approximation. In this case the resonance scattering intensity $I_{S}$ is given by

$$
I_{s}=C I_{0} \omega_{s}^{4} \sum_{\rho \sigma}\left|\left(\alpha_{\rho \sigma}\right)_{f i}\right|^{2} \quad \text { with } \quad\left(\alpha_{\rho \sigma}\right)_{f i}=\sum_{r} \frac{\left\langle f\left|\mu_{\rho}\right| r\right\rangle\left\langle r\left|\mu_{\sigma}\right| i\right\rangle}{\omega_{r i}-\omega_{L}-\mathrm{i} \gamma_{r}}
$$

where $I_{0},\left(\alpha_{\rho \sigma}\right)_{f i}, \mu_{\rho, \sigma}, \omega_{r i}$, and $\gamma_{r}$ are the intensity of the incident light, the transition polarizability, the dipole moment, the transition energy from state $i$ to state $r$, and an electronic damping constant, respectively. Within the adiabatic approximation for the wave functions and a transition dipole moment independent of the vibrational normal coordinates (Albrecht A-term [39]) this results eventually in the relation

$\left(\alpha_{\rho \sigma}\right)_{f i}=A=\sum_{e, \nu_{e}} \frac{\mu_{0 \rho}^{e} \mu_{0 \sigma}^{e}}{\omega_{r i}-\omega_{L}-\mathrm{i} \gamma_{\mathrm{r}}}\left\langle\nu_{f} \mid \nu_{e}\right\rangle\left\langle\nu_{e} \mid \nu_{i}\right\rangle$.

$\mu_{0 \rho, \sigma}^{e}$ are the $\rho, \sigma$ components of the pure electronic transition dipole moments which are assumed to be constant. $r$ stands as abbreviation for the transitions to states $e, \nu_{e}$. The right part of the expression in Eq. (2) depicts the vibronic matrix elements given by the Frank-Condon (FC) integrals. The vibronic quantum numbers $\nu_{i}$ were assumed zero except for $\mathrm{i}=0$. This means all electrons are in the vibronic ground state. $\nu_{f}$ was assumed 1 throughout meaning that only one vibron processes were considered. The experimental results for the 7-AGNR required electronic transitions up to $e=2$, i.e., in solid state terminology $E_{11}$ and $E_{22}$ were needed for the fit and thus experimentally determined. The most relevant parameters for the fit are the transition energies to the first and to the second excited state, the Huang-Rhys factors, and the vibrational frequencies in these states. Values for the transition energies are depicted in Table 2. Excited state frequencies as they are listed in Table 1 were found to be very close to the ground state frequencies. For the resonance of the $\mathrm{CH}$-ipb mode in the case of 7-AGNR the line width in the excited state for the transition $E_{11}$ was considerably smaller than the line width in the ground state. Therefore the peak for the high energy resonance (outgoing resonance) is higher than the peak for the low energy resonance (incoming resonance). All parameters for the fitted resonances are summarized in the Supporting Information Section (f), Tab. S1. The observed transition 

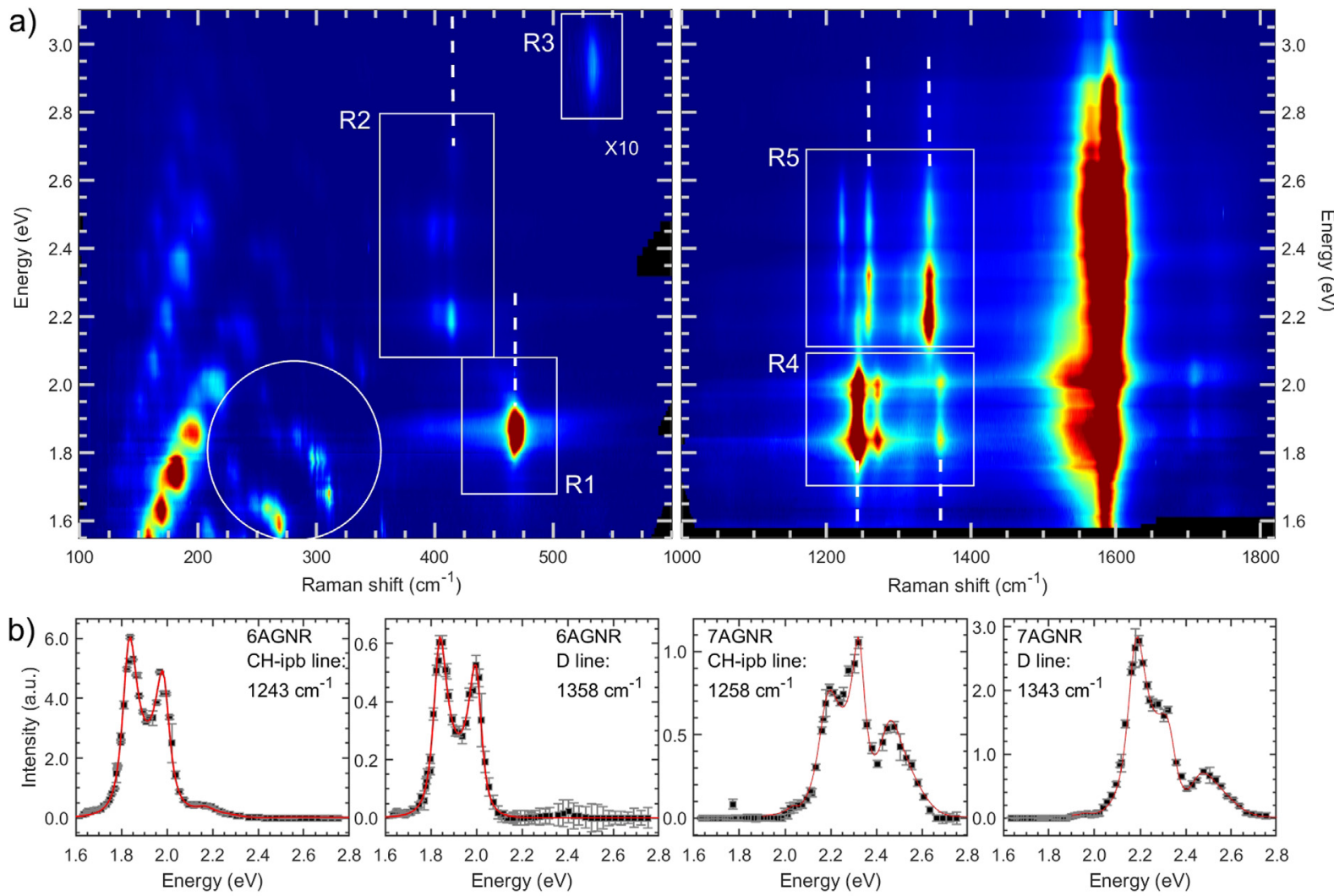

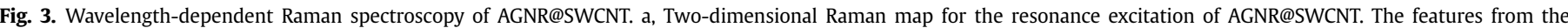

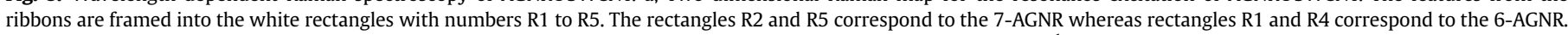

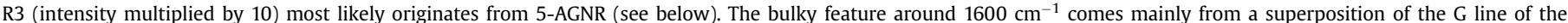

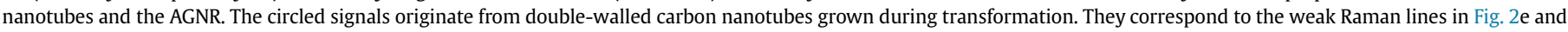

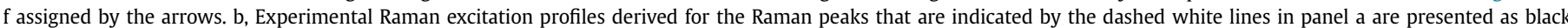

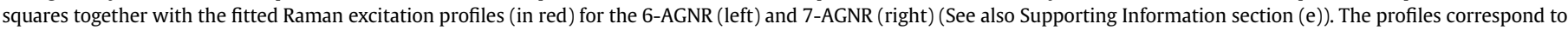
the $\mathrm{CH}$-ipb and $\mathrm{D}$-line, respectively. (A colour version of this figure can be viewed online.)

\section{Table 2}

Transition energies in eV as observed experimentally from resonance Raman analysis, compared to calculated values (in parentheses) and to references for AGNR. Experimental values were rounded to two digits behind the decimal point. Line 1 and line 3 were obtained for 7-AGNR from high frequency modes and from the RBLM, respectively. Column 5 and 6 are from references. The table also shows calculated transition energies for 5-AGNR, together with a value from a reference.

\begin{tabular}{|c|c|c|c|c|c|}
\hline Ribbon & $\begin{array}{l}\mathrm{E}_{11} \\
\text { Exp. } \\
\text { (Calc.) } \\
(\mathrm{eV})\end{array}$ & $\begin{array}{l}\mathrm{E}_{22} \\
\text { Exp. } \\
\text { (Calc.) } \\
(\mathrm{eV})\end{array}$ & $\begin{array}{l}E_{33} \\
(e V)\end{array}$ & $\begin{array}{l}\text { E}_{11} \\
\text { Exp. } \\
\text { (Calc.) } \\
(\mathrm{eV})\end{array}$ & $\begin{array}{l}\mathrm{E}_{22} \\
\text { Exp. } \\
\text { (Calc.) } \\
(\mathrm{eV})\end{array}$ \\
\hline 7-AGNR & $\begin{array}{l}2.18 \\
(2.37)\end{array}$ & $\begin{array}{l}2.45 \\
(2.75)\end{array}$ & (3.03) & $\begin{array}{l}\text { 2.1 Ref. [8] } \\
(1.91)\end{array}$ & $\begin{array}{l}\text { 2.3 Ref. [8] } \\
(2.3)\end{array}$ \\
\hline 7RBLM & 2.18 & 2.43 & & & \\
\hline 6-AGNR & $\begin{array}{l}1.83 \\
(1.82)\end{array}$ & $(3.02)$ & (3.24) & 1.69 Ref. [40] & \\
\hline 5-AGNR & $(0.84)$ & (2.19) & (3.02) & 0.1 Ref. [41] & \\
\hline
\end{tabular}

energies were compared to values calculated by solving the BetheSalpeter equation within the frame of a quasiparticle self-consistent GW calculation. These calculations go beyond the energy dependent Placzek approximation as it was used to derive the Raman spectra described in Fig. 2. The calculation is described in detail in Methods and in Supporting Information Section (b). Table 2 lists the observed transition energies as compared to our calculations and to reported values from optical reflection measurements [8]. The comparison depicted in the table further evidences the successful growth of 7-AGNR and 6-AGNR inside SWCNT.
The two peaks in the resonance for the 6-AGNR represent the transitions to the first and to the second vibronic level in the excited state, or equivalently, the ingoing and outgoing resonance. Higher vibronic levels are neither observed for the 6-AGNR nor for the 7AGNR.

Calculated transition energies for 5-AGNR are included in the table since the values may be relevant for the resonance of the Raman line observed at $533 \mathrm{~cm}^{-1}$ (R3 in Fig. 3) as discussed below. The low value of $0.84 \mathrm{eV}$ calculated for the band gap is consistent with the $3 p+2$ family of these ribbons.

For the analysis of AGNR@SWCNT the RBLMs are particularly important since they exhibit a strong and characteristic response in a frequency region which is free from other Raman lines. In the case of the 7-AGNR@SWCNT the response is unusual since it exhibits several components. Fig. 4a shows a zoomed-in Raman map of region R2. Several peaks can be observed in this region, which become also evident when plotting the Raman spectra for two distinct laser excitations (Fig. 4b). The main peak (highest intensity) appears at a vibrational frequency of $414 \mathrm{~cm}^{-1}$ for $569.5 \mathrm{~nm}$ excitation with an exceptionally small line width of only $7 \mathrm{~cm}^{-1}$. In addition, a shoulder can be observed around $400 \mathrm{~cm}^{-1}$, which is considerably broader $\left(13 \mathrm{~cm}^{-1}\right)$ and exhibits dispersion, i.e. line position shifts with changing excitation energy. Such behavior is well known for the D-line in CNTs but also for conjugated polymers like poly-acetylene [42]. It is an indication for defective structures where vibrational frequencies and electronic transitions exhibit some correlation which eventually leads to photo-selective 

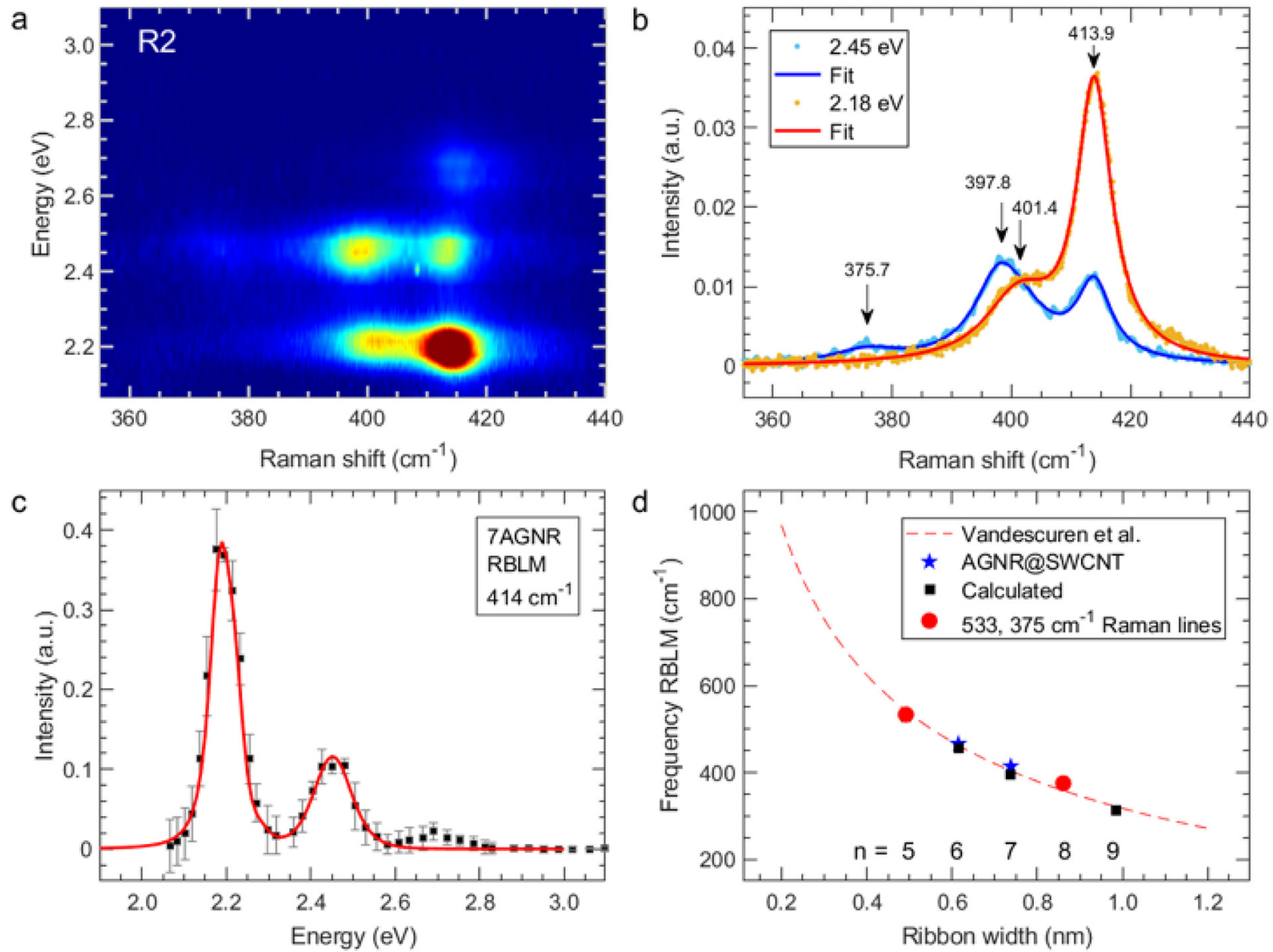

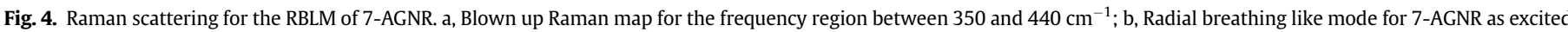

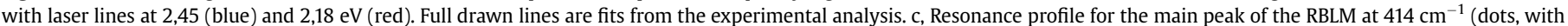

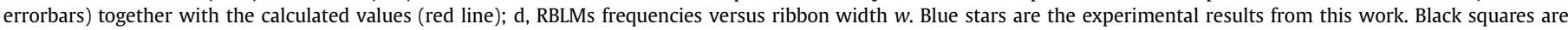

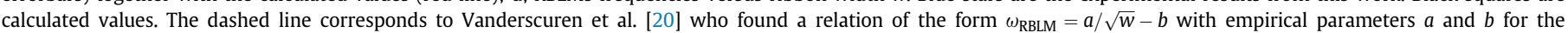

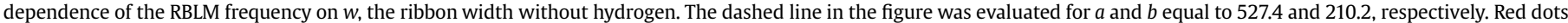
are for the additional Raman lines at 533 and $375 \mathrm{~cm}^{-1}$ if they are assigned to 5-AGNR and 8-AGNR, respectively. The value for $\mathrm{n}=9$ is from a reference. [7].

resonance scattering. In our case such structures may be represented by interfaces between extended or short ribbons of different structure and different topology. In such cases new electronic state inside the gap can be created [17]. Also, modified edges resulting in sample inhomogeneity can lead to slightly modified RBLM-modes and thus result in photo-selective resonance scattering. In addition to the shoulder a very weak Raman signal is observed at $375 \mathrm{~cm}^{-1}$ for $506.1 \mathrm{~nm}$ excitation and at shorter wavelength of $460 \mathrm{~nm}$ a Raman line at $417 \mathrm{~cm}^{-1}$. At even shorter wavelengths of $405 \mathrm{~nm}$ excitation a Raman line is observed at $533 \mathrm{~cm}^{-1}$. (The latter two are not shown in the figure.) The origin of these lines will be discussed below and in some more detail in Supporting Informations Section (e).

The strong line at $414 \mathrm{~cm}^{-1}$ is most appropriate to analyze resonance profiles. This profile is depicted in Fig. 4c. It exhibits two strong resonances and a weak resonance at $2.7 \mathrm{eV}$, which is not statistically significant and originates from a different Raman frequency (see Supporting Information (e)). Due to the low mode energy for the RBLM (51 meV) the two resonances do not explicitly show vibronic splitting. Fitting the strong resonances with the Albrecht A term yields the transition energies listed in Table 2. The resonances have very similar energies as they were observed for the high frequency modes of the 7-AGNR and are therefore assigned to $E_{11}$ and $E_{22}$.
RBLMs for AGNR are known to depend on the width $w$ of the ribbons. In several reports this dependence was observed to follow an $1 / \sqrt{w}$ behavior [20]. A more recent work showed that the RBLM was found to scale with $1 / \mathrm{w}$ [21]. Within the small frequency range one cannot discriminate between the two scaling laws. Fig. 4d depicts this scaling law (Vandescuren diagram) as a dashed line. The black squares are the frequencies calculated here for 5-AGNR, 6-AGNR and 7-AGNR. The experimentally observed frequencies for 6-AGNR and 7-AGNR are indicated as blue stars. The good fit of these values into the Vandescuren diagram is another proof for the observation of such ribbons in our samples. Interestingly the so far unassigned lines at $533 \mathrm{~cm}^{-1}$ and $375 \mathrm{~cm}^{-1}$ fit also very well to the diagram if they are assigned to RBLMs for 5-AGNR and 8-AGNR. Recently the RBLM for 5-AGNR was observed at $533 \mathrm{~cm}^{-1}[12,43]$. However, since both ribbons are narrow gap species of the type $3 p+2, E_{22}$ resonances or even higher transitions must be involved for their observation. Interestingly for 8-AGNR very recently an effective gap of $2.3 \mathrm{eV}$ was reported from STS measurements [44]. This is reasonably close to the $2.45 \mathrm{eV}$ laser used to detect the Raman line at $375 \mathrm{~cm}^{-1}$. The Raman line at $533 \mathrm{~cm}^{-1}$ was observed in our case for excitation with a considerable higher energy than the value calculated for the $E_{22}$ transition. Shortening of the ribbons with respect to infinite long species could be the reason for the enhanced transition energies $[41,45]$ as they are observed here. 


\section{Discussion and conclusion}

\subsection{Discussion}

When using polyaromatic hydrocarbons as precursor molecules (such as coronene [29,30]), the growth of GNRs inside CNTs can be explained by an oligomerization reaction. In our case, however, and similarly as previously reported for tetrathiofulvalene molecules inside CNTs [28], the encapsulated ferrocene molecules first need to be decomposed by the heat treatment and subsequently need to form the nanoribbons. This process is assumed to benefit from the graphene-like walls of the nanotubes, but the exact process is yet unknown. Until now, only graphene nanoribbons with armchair structures were observed, which is probably due to the higher stability of these ribbons. The dominance of the growth of 6AGNR and 7AGNR is due to the mean diameter of the carbon nanotubes used. Ribbons with a higher width would not fit into the tubes without extra stress and too narrow ribbons would be energetically less favorable.

The ribbons observed in our study are definitely hydrogen passivated. This can be concluded from the strong $\mathrm{CH}$-ipb mode observed in the Raman spectra. The strong response of the hydrogen vibration is also reflected in the ab initio calculations. Passivation by more extended hydrocarbon groups is not likely due to the lack of space inside the tubes.

The concentration of the ribbons inside the tubes can be estimated from the available carbon resources and thus the degree of filling of the tubes with ferrocene. A $(17,0)$ tube can be filled with approximately 4 molecules of ferrocene per nm [46], which means $40 \mathrm{C} / \mathrm{nm}$ can be provided. A 7-AGNR needs $33.3 \mathrm{C} / \mathrm{nm}$, hence sufficient carbon atoms are available to form the graphene nanoribbons and thus a high concentration of ribbons can be expected. We indeed observe very high Raman intensities from the AGNRs compared to the Raman signatures of the surrounding CNTs, however, the Raman cross-section of encapsulated AGNRs with respect those of CNTs is not yet known. Hence, one cannot use the Raman intensities to estimate the synthesis yield.

The TEM analysis gives clear evidence for flat objects with ribbon structure inside the tubes. The deviation from a perfect graphenic ribbon structure observed in the figure is a consequence of the electron beam irradiation rather than being intrinsic to the ribbon growth. The identification of the ribbons comes from wavelength-dependent Raman scattering and comparison to high level quantum-chemical calculations. The very narrow Raman lines as depicted in Table 1 and Fig. 2 are evidence for clean and highly unperturbed ribbon material. For the 7-AGNR the experimental and theoretical Raman spectra exhibit unprecedented agreement with respect to frequency of the modes and the relative Raman intensities.

The very sharp and individual peaks of the resonance profiles are evidence for a well-defined edge structure, which does not allow for defect induced modulation and consequently broadening or splitting of the optical transitions.

\subsection{Conclusion}

The results presented above demonstrate that graphene nanoribbons can be grown from thermal decomposition of ferrocene encapsulated in carbon nanotubes. Aberration corrected high resolution transmission electron microscopy provides evidence for the ribbons inside the tubes. Raman scattering combined with first principle calculations and resonance excitation analysis is an excellent tool to identify the structure of GNR even if the ribbons are encapsulated in SWCNTs. Due to its nondestructive nature and frequency selective character the Raman method is superior to any other optical method such as optical absorption or luminescence spectroscopy. In addition the method allows determining the electronic structures of the ribbons, even beyond the HOMO-LUMO gap. The results provide a challenge that ribbons with larger or smaller width than those reported here, can be grown in larger or smaller SWCNTs, respectively, and can be detected with Raman scattering. They thus open a new field in subnanometer graphene ribbon research.

\section{Methods}

Synthesis of GNRs@SWCNT SWCNTs as grown by the eDIPS technique [47] with mean diameter around $1.3 \mathrm{~nm}$ were used as a starting material. All tubes were purified by first etching in air and subsequent treating with $\mathrm{HCl}$ as described previously [48] to remove amorphous carbon and catalytic particles from tube growth. SWCNT bucky paper was obtained by washing and filtering the tubes with distilled water and ethanol.

For the filling process the tubes were first opened by etching in air at $420{ }^{\circ} \mathrm{C}$ and then exposed to ferrocene at $400{ }^{\circ} \mathrm{C}$ for two days in previously evacuated quartz tubes. The transformation of the $\mathrm{FeCp}_{2}$ molecules to the AGNRs inside the tubes was performed by vacuum annealing for several days and at various temperatures as described in the main text. This process resulted in the appearance of new lines in the Raman spectra from some so far unknown objects. Control experiments were performed without opening the tubes but otherwise treating the material identically. In this case almost no new Raman lines were observed as depicted explicitly in the Supporting Information Section (c). This can be considered as evidence for the growth of the objects inside the tubes.

Analysis by Transmission Electron Microscopy To obtain information on the grown objects TEM investigations were performed with a Thermo Fisher (formerly FEI) TITAN G2 80-300 (Fig. $1 \mathrm{a}-\mathrm{e}$ ) at $80 \mathrm{kV}$. Fig. 1f, $\mathrm{g}$ and $\mathrm{i}$ are acquired by the specific Thermo Fisher (formerly FEI) SALVE (subangstrom low voltage electron microscopy) transmission electron microscope fitted with a dedicated chromatic and spherical $(\mathrm{Cc} / \mathrm{Cs})$ aberration corrector developed by CEOS which is fully corrected for the fifth-order axial geometric aberrations (including CS and C5), for the third-order offaxial geometric aberrations, and for the first-order chromatic aberrations (CC), and also equipped a Thermo Fisher Ceta 4K CMOS camera. The exposure time of the images is $1.0 \mathrm{~s}$ and the dose rate is $6.85 * 10^{6}$ electrons $\mathrm{s}^{-1} \mathrm{~nm}^{-2}$.

Theoretical evaluation of vibrational frequencies and modespecific Raman intensities Vibrational frequencies of the ribbons were calculated at the $\Gamma$ point using the Vienna Ab initio Simulation Package (VASP) [49]. Raman intensities $I_{S}$ were evaluated using the frequency dependent Placzek approximation. In this case the intensity of the Raman lines is proportional to the square of the derivative of the frequency dependent polarizability with respect to the phonon normal mode:

$I_{s}\left(\omega_{s}, \omega_{L}\right)=\frac{\omega_{s}^{4}}{\omega_{p h}} \sum_{\rho, \sigma}\left|\frac{\partial \alpha_{\rho, \sigma}\left(\omega_{L}\right)}{\partial Q_{p h}}\right|^{2} \Gamma\left(\omega-\omega_{p h}\right)\left(n\left(\omega_{p h}\right)+1\right)$

where $\alpha_{\rho, \sigma}\left(\omega_{L}\right)$ is the dynamic (frequency dependent) polarizability tensor evaluated from first principle calculations at the laser energy $\omega_{L}$. Calculation of the dynamic polarizability was done within the linear response theory [50] with wave functions and structural parameters used during the frequency calculation and geometrical optimization. $Q_{p h}$ and $\omega_{p h}$ are the phonon normal modes and frequencies, respectively, and $\omega_{S}$ is the frequency of the scattered light. $\omega$ is the difference between $\omega_{L}$ and $\omega_{s} . n\left(\omega_{p h}\right)$ is the Bose-Einstein distribution at room temperature and $\Gamma(x)$ is a normalized 
Lorentzian function with full width at half maximum of $10 \mathrm{~cm}^{-1}$. Numeric derivatives of the polarizability tensor were calculated using symmetric derivatives by manually shifting the atoms according to normal modes in both positive and negative directions. From this the following expression can be obtained for the derivative of the polarizability:

$\frac{\partial \alpha_{\rho, \sigma}\left(\omega_{L}\right)}{\partial Q_{p h}}=\sum_{r, s} \frac{\left\langle f\left|H_{e-p}\right| s\right\rangle\left\langle s\left|H_{e-p h}\right| r\right\rangle\left\langle r\left|H_{e-p}\right| i\right\rangle}{\left(\omega_{L}-\omega_{r}-\mathrm{i} \gamma_{e-p}\right)\left(\omega_{r}-\omega_{s}-\mathrm{i} \gamma_{e-p h}\right)}$

where r,s are intermediate virtual electronic states with energy $\omega_{r, s}$ obtained from first principle calculations, $H_{e-p h}$ (derivative of the electron-ion potential with respect to the normal modes) and $H_{e-p}$ are the Hamiltonians for the electron-phonon and electron-photon coupling, respectively and $\gamma_{e-p h}$ and $\gamma_{e-p}$ are the corresponding damping constants (life times). Details of the derivation can be found in Refs. [32]. The calculation was successfully used previously for the analysis of doping and strain induced changes of the Raman spectra and gap structure of $\mathrm{MoS}_{2}$ [51] and silicene [52].

Optical excitation energies were calculated by solving the Bethe-Salpeter equation within the frame of a quasiparticle selfconsistent GW calculation as built in the QUESTAAL code [53] with ladder diagram corrections [54]. Excitation energies were determined by taking the energy values at the maximum of the peaks present in the imaginary part of the macroscopic dielectric function. Explicitly, calculations were performed for the low gap $3 p+2$ ribbon 5-AGNR, for the moderate gap 3p ribbon 6-AGNR, and for the large gap $3 p+1$ ribbon 7-AGNR. More details on the calculation are in supporting Information Section (b).

Raman scattering Raman spectra were excited at room temperature and at ambient conditions with various lasers in the visible spectral range. The spectra in Fig. 2 were recorded with a Dilor xy800 and with a Labram HR800 microscope. In Fig. 2 spectra with a spectral resolution of less than 2 wavenumbers. Thus the observed linewidths are intrinsic to the ribbon material. Raman intensities were normalized to the $2 \mathrm{D}$ line of the carbon nanotubes, since the 2D-line is known to be highly independent from defects. In the case of resonance analysis normalization was performed to the fundamental Raman line of Si.

Evaluation of Excitation Profile Resonance Raman spectra were evaluated for wavelength dependent excitation in the range between 400 and $800 \mathrm{~nm}$ with $5 \mathrm{~nm}$ step-size. To cover the requested energy range for excitation the following three laser systems were used. 400-526 nm: A frequency-doubled Ti:Sa laser (M Squared SolsTiS external cavity frequency doubled ECD-X module) which was pumped by an $18 \mathrm{~W}$ Sprout-G diode pumped solid state laser (532 nm). 534-605 nm and 610-690 nm: A dye laser (Spectra Physics model 375) pumped by an $\mathrm{Ar}+$ ion laser (Spectra Physics 2020) equipped with either Rhodamine 110 or DCM laser dyes. 690-800 nm: A tunable Ti:Sa laser (Spectra Physics 3900S). The Raman spectra were recorded with a high resolution triple grating Dilor XY800 spectrometer and a liquid nitrogen cooled CCD detector. More details about the recording and evaluation of the Raman spectra can be obtained from Supporting Information Section (e).

\section{Author contributions}

H.K: Supervision, contributed equally to this work, designed and supervised the experiments, analyzed the resonance profiles. L.S: Supervision, contributed equally to this work, designed and supervised the experiments, prepared the samples and did the characterization with spontaneous Raman scattering in Vienna with lasers at wavelength of 633 and $568 \mathrm{~nm}$. S.C: Formal analysis, performed the wavelength-dependent resonance Raman scattering experiments and their analysis. M.M: Formal analysis, performed the wavelength-dependent resonance Raman scattering experiments and their analysis. W.W: Formal analysis, performed the wavelength-dependent resonance Raman scattering experiments and their analysis. K.C: performed HRTEM characterization and simulations. U.K: performed HRTEM characterization and simulations. J.K: performed the first principles DFT calculations. J.K: performed the first principles DFT calculations. G.K: performed the first principles DFT calculations. T.S: provided the SWCNTs. T.P: provided the laboratory facilities and the Raman setup in Vienna. All authors discussed the results and commented on the manuscript at all stages.

\section{Declaration of competing interest}

The authors declare that they have no known competing financial interests or personal relationships that could have appeared to influence the work reported in this paper.

\section{Acknowledgement}

Work supported by the NSFC (51902353), the FWF project P21333-N20, and the NKFIH, Grant No. K-115608. L.S. acknowledges the financial support from the Natural Science Foundation of Guangdong Province (Grant No. 2019A1515011227) and the Sun Yat-Sen University (Grant No. 29000-18841218, 29000-31610028) J.K., J.K. and G.K. further acknowledge the [NIIF] for awarding access to resource based in Hungary at Debrecen, they further acknowledge support by the National Research Development and Innovation Office of Hungary within the Quantum Technology National Excellence Program (Project No. 2017-1.2.1-NKP-2017-00001), and the ELTE Excellence Program (1783-3/2018/FEKUTSTRAT) supported by the Hungarian Ministry of Human Capacities. K.C. acknowledges the China Scholarship Council (CSC) for financial support. U.K. acknowledges the support of the Graphene Flagship and DFG SPP Graphene as well as the DFG and the Ministry of Science, Research and the Arts (MWK) of Baden-Wuerttemberg within the frame of the SALVE project. M.M., S.C., and W.W. acknowledge funding from the Fund for Scientific Research Flanders (FWO projects No. G040011 N, G02112 N, G035918 N, G036618 $\mathrm{N}$ and the EOS-charming project G0F6218 N [EOS-ID 30467715]). M.M. acknowledges funding of a DOCPRO4 PhD scholarship from the UAntwerp research fund (BOF) and S.C. also acknowledges funding from the European Research Council Starting Grant No. 679841.

\section{Appendix A. Supplementary data}

Supplementary data to this article can be found online at https://doi.org/10.1016/j.carbon.2020.08.065.

\section{References}

[1] P.B. Bennett, et al., Bottom-up graphene nanoribbon field-effect transistors, Appl. Phys. Lett. 103 (2013), 253114.

[2] L. Martini, Graphene nanoribbon devices, Carbon 146 (2019) 36-42.

[3] K.S. Novoselov, et al., Electric field effect in atomically thin carbon films, Science 306 (2004) 666-669.

[4] Y.W. Son, M.L. Cohen, S.G. Louie, Energy gaps in graphene nanoribbons, Phys. Rev. Lett. 97 (2006) 216803.

[5] V. Barone, O. Hod, G.E. Scuseria, Electronic structure and stability of semiconducting graphene nanoribbons, Nano Lett. 6 (2006) 2748-2754.

[6] J. Cai, et al., Atomically precise bottom-up fabrication of graphene nanoribbons, Nature 466 (2010) 470-473.

[7] L. Talirz, P. Ruffieux, R. Fasel, On-surface synthesis of atomically precise graphene nanoribbons, Adv. Mater. 28 (2016) 6222-6231. 
[8] R. Denk, et al., Exciton-dominated optical response of ultra-narrow graphene nanoribbons, Nat. Commun. 5 (2014) 4253.

[9] R.A. Durr, et al., Orbitally matched edge-doping in graphene nanoribbons J. Am. Chem. Soc. 140 (2018) 807-813.

[10] Q. Sun, et al., Massive Dirac fermion behavior in a low bandgapgraphene nanoribbon near a topological phase boundary, Adv. Mater. 32 (2020) 1906054.

[11] L. Gigli, et al., Detachment dynamics of graphene nanoribbons on gold, ACS Nano 13 (2019) 689-697.

[12] J. Overbeck, et al., Optimized substrates and measurement approaches for Raman spectroscopy of graphene nanoribbons, Phys. Status Solidi B (2019), 1900343.

[13] I.A. Verbitskiy, et al., Raman fingerprints of atomically precise graphene nanoribbons, Nano Lett. 16 (2016) 3442-3447.

[14] Z. Pedramrazi, et al., Concentration dependence of dopant electronic structure in bottom-up graphene nanoribbons, Nano Lett. 18 (2018) 3550-3556.

[15] A. Narita, Z.P. Chen, Q. Chen, K. Mullen, Solution and on-surface synthesis of structurally defined graphene nanoribbons as a new family of semiconductors, Chem. Sci. 10 (2019) 964-975.

[16] Y.C. Chen, et al., Molecular bandgap engineering of bottom-up synthesized graphene nanoribbon heterojunctions, Nat. Nanotechnol. 10 (2015) 156-160.

[17] T. Ca, F. Zhao, S.G. Louie, Topological phases in graphene nanoribbons: junction states, spin centers, and quantum spin chains, Phys. Rev. Lett. 119 (2017), 076401.

[18] O. Groning, et al., Engineering of robust topological quantum phases in graphene nanoribbons, Nature 560 (2018) 209-213.

[19] R. Akilimali, et al., Graphene nanoribbon-tio2-quantum dots hybrid photoanode to boost the performance of photoelectrochemical for hydrogen generation, Catal. Today 340 (2020) 161-169.

[20] M. Vandescuren, P. Hermet, V. Meunier, L. Henrard, P. Lambin, Theoretica study of the vibrational edge modes in graphene nanoribbons, Phys. Rev. B 78 (2008), 195401.

[21] D. Liu, C. Daniels, V. Meunier, A.G. Every, D. Tománek, In-plane breathing and shear modes in low-dimensional nanostructures, Carbon 157 (2020) $364-370$.

[22] H.E. Lim, et al., Growth of carbon nanotubes via twisted graphene nanoribbons, Nat. Commun. 4 (2013) 2548.

[23] A.N. Khlobystov, Carbon nanotubes: from nano test tube to nano-reactor, ACS Nano 5 (2011) 9306-9312.

[24] H. Kuzmany, et al., The growth of new extended carbon nanophases from ferrocene inside single-walled carbon nanotubes, Phys. Stat. Sol. RRL 11 (2017) 1700158.

[25] R. Kitaura, et al., High-yield synthesis of ultrathin metal nanowires in carbon nanotubes, Angew. Chem. Int. Ed. 48 (2009) 8298-8302.

[26] L. Shi, et al., Confined linear carbon chains as a route to bulk carbyne, Nat. Mater. 15 (2016) 634-639.

[27] T. Pham, et al., Torsional instability in the single-chain limit of a transition metal trichalcogenide, Science 361 (2018) 263-266.

[28] T.W. Chamberlain, et al., Size, structure, and helical twist of graphene nanoribbons controlled by confinement in carbon nanotubes, ACS Nano 6 (2012) 3943-3953.

[29] A.I. Chernov, et al., Optical properties of graphene nanoribbons encapsulated in single-walled carbon nanotubes, ACS Nano 7 (2013) 6346-6353.

[30] I.V. Anoshkin, et al., Coronene encapsulation in single-walled carbon nanotubes: stacked columns, peapods, and nanoribbons, ChemPhysChem 15 (2014) 1660-1665.

[31] H.E. Lim, et al., Fabrication and optical probing of highly extended, ultrathin graphene nanoribbons in carbon nanotubes, ACS Nano 9 (2015) 5034-5040.
[32] G. Kukucska, V. Zólyomi, J. Koltai, Frequency-dependent Ab Initio Resonance Raman Spectroscopy, 2019 arXiv preprint arXiv:1903.00253.

[33] A. Chuvilin, et al., Self-assembly of a sulphur-terminated graphene nanoribbon within a single-walled carbon nanotube, Nat. Mater. 10 (2011) 687-692.

[34] H. Kuzmany, L. Shi, T. Pichler, The origin of nondispersive Raman lines in the d-band region for ferrocene@hipco swcnts transformed at high temperatures, Phys. Status Solidi B 252 (2015) 2530-2535.

[35] G. Borin Barin, et al., Substrate transfer and ex situ characterization of onsurface synthesized graphene nanoribbons, ACS Appl. Nano Mater. 2 (2019) 2184-2192.

[36] A. Basagni, et al., Molecules-oligomers-nanowires-graphene nanoribbons: a bottom-up stepwise on-surface covalent synthesis preserving long-range order, J. Am. Chem. Soc. 137 (2015) 1802-1808.

[37] W. Kiefer, Nonlinear Raman Spectroscopy Infrared and Raman Spectroscopy Ed B Schrader, 1995.

[38] E.H. Haroz, et al., Resonant Raman spectroscopy of armchair carbon nanotubes: absence of broad G- feature, Phys. Rev. B 84 (2011), 121403.

[39] J. Tang, A. Albrecht, Developments in the theories of vibrational Raman intensities, in: Raman Spectroscopy, vols. 33-68, Springer, 1970.

[40] N. Merino-Diez, et al., Width-dependent band gap in armchair graphene nanoribbons reveals fermi level pinning on Au(111), ACS Nano 11 (2017) $11661-11668$

[41] A. Kimouche, et al., Ultra-narrow metallic armchair graphene nanoribbons, Nat. Commun. 6 (2015) 10177.

[42] H. Kuzmany, E.A. Imhoff, D.B. Fitchen, A. Sarhangi, Frank-condon approach for optical-absorption and resonance Raman-scattering in trans-polyacetylene, Phys. Rev. B 26 (1982) 7109-7112.

[43] Z.P. Chen, et al., Lateral fusion of chemical vapor deposited $\mathrm{N}=5$ armchair graphene nanoribbons, J. Am. Chem. Soc. 139 (2017) 9483-9486.

[44] K. Sun, P. Ji, On-surface synthesis of 8 - and 10-armchair graphene nanoribbons, Small 15 (2019), 1804526.

[45] L. Talirz, H. Sde, S. Kawai, Band gap of atomically precise graphene nanoribbons as a function of ribbon length and termination, ChemPhysChem 20 (2019) 2348-2353.

[46] W. Plank, et al., Electronic structure of carbon nanotubes with ultrahigh curvature, ACS Nano 4 (2010) 4515-4522.

[47] T. Saito, et al., Selective diameter control of single-walled carbon nanotubes in the gas-phase synthesis, J. Nanosci. Nanotechnol. 8 (2008) 6153-6157.

[48] H. Kuzmany, et al., Nondispersive Raman lines in the d-band region for ferrocene functionalized carbon nanotubes, Phys. Status Solidi B 251 (2014) 2457-2460.

[49] G. Kresse, J. Furthmuller, Efficient iterative schemes for ab-initio total-energy calculations using a plane-wave basis set, Phys. Rev. B 54 (1996) 11169-11186.

[50] M. Gajdos, K. Hummer, G. Kresse, J. Furthmuller, F. Bechstedt, Linear optical properties in the projector-augmented wave methodology, Phys. Rev. B 73 (2006), 045112.

[51] J. Petö, et al., Moderate strain induced indirect bandgap and conduction electrons in mos 2 single layers, npj 2D Mater. Appl. 3 (2019) 1-6.

[52] G. Kukucska, V. Zolyomi, J. Koltai, Characterization of epitaxial silicene with Raman spectroscopy, Phys. Rev. B 98 (2018), 075437.

[53] T. Kotani, M. van Schilfgaarde, Quasiparticle self-consistent gw method: a basis for the independent-particle approximation, Phys. Rev. B 76 (2007), 165106.

[54] B. Cunningham, M. Gruning, P. Azarhoosh, D. Pashov, M. van Schilfgaarde, Effect of ladder diagrams on optical absorption spectra in a quasiparticle selfconsistent gw framework, Phys. Rev. Materials 2 (2018), 034603. 